\title{
Diabetic Pregnancies in Yeovil, Somerset; Management and Outcomes
}

\author{
Madu AE* \\ Department of Obstetrics and Gynaecology, Yeovil, \\ Somerset, United Kingdom \\ *Correspondling author: Madu AE, Acting Specialist \\ Registrar, Obstetrics and Gynaecology Yeovil Hospital \\ NHS Foundation Trust, Yeovil, Somerset, United \\ Kingdom
}

Received: May 04, 2017; Accepted: May 23, 2017; Published: May 30, 2017

\begin{abstract}
Aim and Objectives: The aim was to assess the management and outcomes of diabetic pregnancies in 2005; highlight areas of poor performance and recommend ways to improve the outcomes in those areas highlighted.
\end{abstract}

Methods: A pre-audit assessment was performed to assess what areas needed auditing. The last audit in this important area in medical obstetrics was performed in 2005 for diabetic pregnancies seen in 2002 which was about 5 years before the present study. Audit pro forma was prepared and approved.

Extensive literature searches were performed to assess what the standards were, including Confidential Enquiry into Maternal and Child Health (CEMACH) Reports 2002-3 and 2005, Scottish Intercollegiate Guidelines Network, evidence based Oxford Handbook of Obstetrics and Gynaecology, etc.

The previous audit titled "Management of Diabetes in Pregnancy for 2002" and the local protocol for managing diabetic pregnancies were reviewed prior to commencing this study.

The electronic data on the diabetic pregnancies in 2005 was collated with the help of the Clinical Audit Department.

There was systematic review of the hospital case notes of these women. These case notes were reviewed thrice to ensure accuracy and consistency of the findings or results. There was close cooperation with the Clinical Audit department at various stages of evolution of the audit.

Results/Conclusion: In 2005, there were a total of 1404 live births and 27 of there were diabetic pregnancies, accounting for an incidence of diabetic pregnancies of $1.92 \%(27 / 1404)$. The incidence of type 1 diabetes in pregnancy was $0.5 \%(7 / 1404)$ (cf national average of $0.5 \%$ ), while the incidence of GDM was $1.35 \%(19 / 1404)$.

There were other important findings in relation to the management and outcomes of diabetic pregnancy stated in the study.

Keywords: Diabetic pregnancies; Yeovil District Hospital; Medical complications of pregnancy

\section{Abbreviations}

WHO: World Health Organisation; GTT: Glucose Tolerance Test; ELCS: Elective Lower Segment Caesarean Section; EMCS: Emergency Lower Segment Caesarean Section; PPH: Postpartum Haemorrhage; IGT: Impaired Glucose Tolerance Test; GDM: Gestational Diabetes Mellitus; HbA1c: Glycosylated Haemoglobin 1c; CTG: Cardiotocograph; ANC: Antenatal Care; RPOC: Retained Products of Conception; BMI: Body Mass Index

\section{Introduction}

Pregnancy is a state of insulin resistance due to anti-insulin, diabetogenic hormones such as human placental lactogen, cortisol, oestrogen, progesterone and glucagon, produced by the placenta. Pregnancy therefore alters glucose metabolism. Compared to the non-pregnant state, the fasting glucose levels are reduced while the postprandial levels are elevated. Insulin production is increased two folds in pregnancy while the insulin requirement increases throughout pregnancy and is maximal at term. Glycosuria also increases due to the lowering of renal threshold for glucose. The prevalence of type 1 and type 2 diabetes is $0.5 \%$ and $2 \%$ respectively. A more recent literature, [1], stated that established diabetes affect $1-2 \%$ of pregnancies. For Asian communities, the prevalence of type 2 is $10 \%$ [2]. The same can be said for Arab communities. This is a five or more-fold increase in these communities compared to other communities. This is attributed to increase in fasting blood sugar due to consanguinity $[3,4]$.

Gestational diabetes is said to exist when the fasting glucose level is more than or equal to $7.0 \mathrm{mmol} / \mathrm{L}$ or when the 2 hour glucose is greater than or equal to $11.0 \mathrm{mmol} / \mathrm{L}$. Women with Impaired Glucose Tolerance (IGT) have normal fasting blood glucose when 2 hour glucose $>7.8 \mathrm{mmol} / \mathrm{L}$ or $<11 \mathrm{mmol} / \mathrm{L}$. Only one of these two values needs to be abnormal to make a diagnosis. Currently, the 
World Health Organisation (WHO) includes gestational IGT with gestational diabetes according to [1]. The latter went on to state that a number of women diagnosed in pregnancy have previously undetected type 1 or 2 diabetes (20-30\%). Despite this fact, WHO still does not advocate universal screening but rather a screening based on identified risk factors.

The study of diabetes in pregnancy is important because of the effects of diabetes on the pregnancy, the effects of pregnancy on diabetes and complications (maternal, fetal and neonatal) of diabetes in pregnancy.

\section{Effects of pregnancy on diabetes}

(a) Reversible deterioration of proteinuria and renal function. This nephropathy occurs in 5-10\% of women with increased risk of pre-eclampsia and fetal growth restriction in this group of women, thus making increased maternal and fetal surveillance necessary.

(b) Development or worsening of existing retinopathy especially with rapid improvement of glycaemic control. This rapid improvement of glycaemic control, leads to increased retinal blood flow which can cause retinopathy. There is a two-fold increase in risk of development or progression of existing retinal disease. Early changes usually revert to normal after delivery. However, it is important that all women with diabetes have assessments for retinopathy while pregnant and that those with proliferative retinopathy, be treated.

(c) Increased incidence of hypoglycaemia episodes with tighter glycaemic control.

(d) Rare ketoacidosis associated with infection, hyperemesis, steroid therapy or beta-sympathomimetic tocolytic therapy.

(e) Ischaemic heart disease; pregnancy is known to increase cardiac load so that women with pre-existing cardiac disease need to have cardiac status assessment by a cardiologist even before pregnancy.

\section{Effects of diabetes on pregnancy}

Maternal hyperglycemia leads to fetal hyperglycemia, the latter through fetal beta-pancreatic cell hyperplasia which in turn leads to fetal hyperinsulinemia. The latter promotes fetal growth leading to macrosomia, organomegaly, fetal polyuria causing polyhydramnios, and increased fetal erythropoiesis. The hyperinsulinemic fetus has neonatal hypoglycaemia after delivery because of the withdrawal of the stimulating placental hormones when the placenta is removed after the birth of the baby.

\section{Other effects of diabetes on pregnancy are summarised below}

(a) Congenital anomalies; sacral agenesis, cardiac, skeletal and neural tube defects. If the $\mathrm{HbA} 1 \mathrm{c}$ is less than $8 \%$, the risk of congenital defect is $5 \%$ but if more than $8 \%$, the risk increases to $25 \%$ and is also associated with increased risk of miscarriage.

(b) Perinatal and neonatal mortality is 2-4 times higher, unexplained fetal death and fetal growth restriction can also occur.

(c) Incidence of fetal macrosomia associated with polyhydramnios; preterm rupture of membranes fetal polyuria; risk of shoulder dystocia and operative deliveries are all increased. (d) Delayed fetal surfactant production due to reduced production of fetal pulmonary phospholipids,

(f) Fetal jaundice, hypocalcaemia, hypomagnesaemia, hypothermia and polycythaemia

(f) Increased risk of pre-eclampsia especially if there in underlying renal or vascular problem [1], recommended a multi-disciplinary approach involving the following practitioners; obstetrician, physician/diabetologist, dietician and diabetic specialist nurse/ midwife.

\section{Complications of diabetes in pregnancy}

Fetal complications are: preterm labour; polyhydramnios and macrosomia increased by $25 \%$ and $25-40 \%$ respectively [1]; miscarriage in diabetes with poor controls; congenital malformations in diabetes with poor control (neural tube defects, microcephaly, cardiac and renal malformations and sacral agenesis). Fetal growth restriction and unexplained still birth can also occur.

Neonatal complications are: Respiratory distress syndrome and birth trauma like shoulder dystocia, fractures, asphyxia, and Erb's palsy. There may be biochemical derangements namely hypoglycaemia, hypocalcaemia and hypomagnesaemia. Other complications are; polycythaemia, jaundice, hypothermia and cardiomegaly.

Maternal complications are: urinary tract infections; recurrent vulvovaginal candidiasis; pre-eclampsia and pregnancy induced hypertension; obstructed labour; operative deliveries; increased operative vaginal deliveries and caesarean section, increase in retinopathy by $15 \%$ [1]; increase in nephropathy and cardiac disease.

\section{Aims and Objectives}

(a) To assess if the management of these women for 2005 was in line with known evidence based guidelines and to highlight areas of poor management.

(b) To highlight the unfavourable outcomes and offer suggestions for an improved outcome.

(c) To calculate the prevalence and incidence of diabetic pregnancies in the hospital.

(d) To make recommendations that may influence future management of these patients.

(e) To complete the study by late 2006 and present it in early 2007.

\section{Materials and Methods}

(a) A pre-audit assessment was performed to assess what areas needed auditing in consultation with other relevant staff like the Medical Obstetric Lead and Midwives.

(b) This audit was chosen following assessment of potential areas to audit and it impact on the practice in the unit. It was concluded that this audit would be more useful to the department due to the prevalence of diabetes in pregnancy.

(c) Submission and approval of audit proposal was then made and Medical Records request form was completed.

(d) I reviewed of the previous audit on Management of Diabetes 
in pregnancy for 2002 and also the local protocol for managing a diabetic pregnancy.

(e) Literature search for standards of practice was performed and the following were collated; CEMACH Reports of 2002-3 and 2005, Scottish intercollegiate guidelines Network, Evidence based Oxford Handbook of Obstetrics and Gynaecology [6-8].

The Green Top guideline on diabetic pregnancies was scheduled to be published later in November 2007.

(f) The audit pro forma was prepared, late amended and later submitted for ethical and departmental approval.

(g) Collation of electronic data (Yeovil District Hospital Maternity Information) on the diabetic pregnancies for 2005 was conducted with the help of the clinical audit department.

(h) Systematic and repeated reviews of the hospital case noted of these women.

These reviews were performed thrice to ensure accuracy and consistency of the results.

(i) There was close cooperation with the Clinical audit department at various stages of the audit.

Standards (see standards, and pro forma annexed as supplementary material) were:

(1) Contraception and Pre-pregnancy care by multidisciplinary team.

(2) Nutrition management before, during and after pregnancy.

(3) Optimization of glycaemic control by various methods stated in the pro forma.

(4) Multidisciplinary care during and after pregnancy.

(5) Preventing complications.

Obstetric; birth trauma, pre-eclampsia, shoulder dystocia, operative delivery etc

Metabolic; hyperglycaemia, hypoglycaemia, diabetic ketoacidosis etc

Microvascular (renal, retinal)

(6) Fetal monitoring/surveillance as indicated.

(7) Delivery; the timing to be individualized. Elective caesarean section if estimated fetal weight is more than $4.5 \mathrm{~kg}$

(8) For GDM; repeating GTT 6weeks postpartum to assess glucose tolerance after pregnancy.

\section{Analysis of Findings}

Total births for 2005 were 1404; total number of diabetic pregnancies were 27 , the study population; incidence of diabetic pregnancies was $1.92 \%$ (27/1404); incidence of type 1 diabetes in pregnancy was $0.5 \%(7 / 1404)$ (cf national average of $0.5 \%)$, while the incidence of GDM was 1.35\% (19/1404).

+Some babies had more than one unfavourable outcome.

+ No adverse fetal or maternal outcomes in 7 women.
Table 1: Type of diabetic pregnancies.

\begin{tabular}{|c|c|}
\hline Type 1 & 7 \\
\hline Type 2 & $0 *$ \\
\hline GDM (diet controlled) & 5 \\
\hline GDM (insulin controlled) & 14 \\
\hline Impaired tolerance & 1 \\
\hline
\end{tabular}

*No case of Type 2 diabetes seen.

Table 2: Age groups.

\begin{tabular}{|c|c|}
\hline$<20$ & 2 \\
\hline $20-25$ & 6 \\
\hline $26-30$ & 11 \\
\hline $31-35$ & 6 \\
\hline $36-40$ & 2 \\
\hline
\end{tabular}

Table 3: Gravidity

\begin{tabular}{|c|c|}
\hline 1 & 6 \\
\hline 2 & 8 \\
\hline 3 & 7 \\
\hline 4 & 2 \\
\hline$>4$ & 4 \\
\hline
\end{tabular}

Table 4: Parity.

\begin{tabular}{|c|c|}
\hline 0 & 10 \\
\hline 1 & 13 \\
\hline 2 & 4 \\
\hline 3 & 0 \\
\hline 4 & 0 \\
\hline
\end{tabular}

Table 5: Preconception counselling (type 1,2, previous GDM).

\begin{tabular}{|c|c|}
\hline yes & 1 \\
\hline No & 9 \\
\hline total & 10 \\
\hline
\end{tabular}

Table 6: Preconception folic acid (type 1,2).

\begin{tabular}{|c|c|}
\hline yes & 0 \\
\hline no & 7 \\
\hline total & 7 \\
\hline
\end{tabular}

\section{D is c u s s i n / Critique/ Comparing Performance with Targets}

From the data stated, it can be seen that for 2005, there was no patient with type 2 diabetes (Table 1 ) despite the latter being four times more common compared with type 1 diabetes.

Most of these women are in the age group of 26-30 (Table 2) and most of them were primigravidas (Tables 3,4).

Despite the importance of preconception counselling, only 1 patient had a documented evidence of having had this counselling (Table 5) and none of the patients with type 1 and 2 diabetes had preconception folic acid (Table 6).

Of the 7 women that were eligible for preconception $\mathrm{HbAlc}$ test, only one had it done (Table 7). 
Table 7: Preconception hba $\mathrm{hc}_{1 \mathrm{c}}$ (type 1 and 2).

\begin{tabular}{|c|c|}
\hline Done & 1 \\
\hline Not done & 6 \\
\hline Total & 7 \\
\hline
\end{tabular}

Table 8: Post conception folic acid.

\begin{tabular}{|c|c|}
\hline Yes & 21 \\
\hline No & 6 \\
\hline Total & 17 \\
\hline
\end{tabular}

Table 9: Planned pregnancy (type 1, 2, previous GDM).

\begin{tabular}{|c|c|}
\hline Yes & 7 \\
\hline No & 3 \\
\hline Total & 10 \\
\hline
\end{tabular}

Table 10: Gestational age at booking

\begin{tabular}{|c|c|}
\hline First trimester & 26 \\
\hline Second trimester & 1 \\
\hline Third trimester & 0 \\
\hline
\end{tabular}

Table 11: Types of care.

\begin{tabular}{|c|c|}
\hline Shared care & 26 \\
\hline GP led care & 0 \\
\hline Community midwife led care & 1 \\
\hline
\end{tabular}

1 woman under a community midwife was transferred to shared care at 26 weeks gestation.

Table 12: Body Mass Index (BMI) at booking.

\begin{tabular}{|c|c|}
\hline$<20$ & 0 \\
\hline $21-25$ & 8 \\
\hline $26-29$ & 8 \\
\hline $30-35$ & 5 \\
\hline $36-30$ & 5 \\
\hline$>40$ & 1 \\
\hline
\end{tabular}

Table 13: Gestational age at GTT (for GDM and impaired glucose tolerance).

\begin{tabular}{|c|c|}
\hline Before 28 weeks & 5 \\
\hline At 28 weeks & 6 \\
\hline Beyond 28 weeks & 9 \\
\hline total & 20 \\
\hline
\end{tabular}

GTT performed for 20 women (1 IGT, 19 GDM).

In 7 women pregnancy was planned (Table 8-10)

Most women (26) had booked for antenatal care in first trimester while 1 booked in the second trimester. Most of the women (26) had shared care (Table 11).

Most of the women reviewed had BMI 21-25(8) and 26-29(8) (Table 12)

A record number of the women (9) who had GDM had it diagnosed after 28weeks gestation while 6 had it diagnosed at 28 weeks gestation (Table 13).

None of the pregnancies were twins (Table 14,15).
Antenatal Events

Table 14: Number of fetuses in each pregnancy.

\begin{tabular}{|c|c|}
\hline singletons & 27 \\
\hline twins & 0 \\
\hline triplets & 0 \\
\hline
\end{tabular}

Table 15: Antenatal visits (cmw/hospital).

\begin{tabular}{|c|c|}
\hline$<10$ times & 0 \\
\hline $10-15$ times & 16 \\
\hline $16-20$ times & 10 \\
\hline$>20$ times & 1 \\
\hline
\end{tabular}

Many of the antenatal visits were for CTG monitoring from 36wks.

Table 16: Duration of hospital admissions.

\begin{tabular}{|c|c|}
\hline$<5$ times & 15 \\
\hline $5-10$ times & 11 \\
\hline$>10$ times & 1 \\
\hline
\end{tabular}

Table 17: Fetal surveillance.

\begin{tabular}{|c|c|}
\hline Downs screening & 20 \\
\hline AFP alone & 1 \\
\hline Declined Downs screening & 6 \\
\hline Scan at 20weeks gestation & 27 \\
\hline Scan at 22-24weeks gestation & 3 \\
\hline
\end{tabular}

Table 18: Abdominal circumference (ac) on last scan.

\begin{tabular}{|c|c|}
\hline$<5^{\text {th }}$ centile & 0 \\
\hline $5-50^{\text {th }}$ centile & 0 \\
\hline $50^{\text {th }}$ centile & 3 \\
\hline $95^{\text {th }}$ centile & $14^{\star}$ \\
\hline$>95^{\text {th }}$ centile & $10^{\star}$ \\
\hline
\end{tabular}

Table 19: Diabetic control (for type 1,2 and GDM on insulin) (HBA ${ }_{1} \mathrm{C} 4$ weekly).

\begin{tabular}{|c|c|}
\hline $1-4$ weeks & $0^{*}$ \\
\hline $5-8$ weeks & 5 \\
\hline $9-12$ weeks & 0 \\
\hline once & 4 \\
\hline twice & 3 \\
\hline Few times/irregular & 1 \\
\hline none & 8 \\
\hline total & 21 \\
\hline
\end{tabular}

In table 15, it can be seen that a record number of 16 women had visited a member of the shared team 10-15 times, while 10 women had antenatal visits 16-20 times. Table 16, shows that11 women had admissions 5-10 times.

20 women had Down's screening while 7 declined, 3 women had repeat scan at 22-24weeks gestation (Table 17).

In Table 18,14 fetuses had their growth at the $95^{\text {th }}$ centile while 10 fetuses had it at $>95^{\text {th }}$ centile consistent with what we already know about the weight of babies of diabetic mothers.

In Tables 19-21, it can be seen that surveillance of diabetic control 
Table 20: Renal function test for type 1, 2 and GDM on insulin (3 weekly).

\begin{tabular}{|c|c|}
\hline $1-3$ weeks & $0^{*}$ \\
\hline $4-6$ weeks & 0 \\
\hline $7-9$ weeks & 2 \\
\hline $10-12$ weeks & 3 \\
\hline once & 5 \\
\hline twice & 5 \\
\hline Few times/irregular & 5 \\
\hline none & 1 \\
\hline total & 21 \\
\hline
\end{tabular}

Table 21: Eye examination (preconception, $1^{\text {st }}, 2^{\text {nd }}$ and $3^{\text {rd }}$ trimester).

\begin{tabular}{|c|c|}
\hline Preconception & \\
\hline $1^{\text {st }}$ & 1(type 1) \\
\hline $2^{\text {nd }}$ & 0 \\
\hline $3^{\text {rd }}$ & 0 \\
\hline none & 20 \\
\hline
\end{tabular}

Table 22: Gestation (weeks) at delivery.

\begin{tabular}{|c|c|}
\hline$<37$ & $1 / 27$ \\
\hline $37-40$ & $26 / 27^{\star}$ \\
\hline$>40$ & 0 \\
\hline
\end{tabular}

Table 23: Other antenatal events.

\begin{tabular}{|c|c|}
\hline Steroid use & $5 / 27$ \\
\hline Spontaneous miscarriage & 0 \\
\hline Polyhydramnios & $1 / 27^{\star}$ \\
\hline Preterm delivery & 0 \\
\hline
\end{tabular}

Table 24: Adverse antenatal outcomes.

\begin{tabular}{|c|c|}
\hline Diabetic ketoacidosis & 1 \\
\hline Persistent UTI, anaemia in pregnancy/collapse/fainting & 1 \\
\hline Breech presentation & 1 \\
\hline Hyperemesis/UTI & 1 \\
\hline Haematuria/UTI & 1 \\
\hline UTI/itching in pregnancy & 1 \\
\hline Obstetric cholestasis & 1 \\
\hline None & 21 \\
\hline
\end{tabular}

was very poor and was not performed in line with the guidelines or acceptable practice. There was no consistency in approach and timing of surveillance aimed at preventing or reducing maternal and fetal complication or maternal end organ damage.

Most women were delivered between 37-40weeks gestation (Table 22), 1 had mild polyhydramnios and 5 had steroids (Table 23).

Adverse maternal antenatal outcomes are highlighted in Table 24.

Tables 25,26 describes the onset and duration of labour for the 13 women who laboured.

14.8\% (4) had EMCS, 44\% (12) had ELCS and 1 had a scheduled caesarean section, see Table 27 . These findings were comparable
Labour Events

Table 25: Onset of labour.

\begin{tabular}{|c|c|}
\hline Spontaneous & 2 \\
\hline induced & 5 \\
\hline Induced \&augmented & 4 \\
\hline Spontaneous and augmented & 2 \\
\hline total & 13 \\
\hline
\end{tabular}

Table 26: Duration of labour (hours).

\begin{tabular}{|c|c|}
\hline$<10$ & 9 \\
\hline$>10$ & 1 \\
\hline total & 10 \\
\hline
\end{tabular}

Table 27: Mode of delivery.

\begin{tabular}{|c|c|}
\hline Normal vaginal & 9 \\
\hline Ventouse & 1 \\
\hline Forceps & 0 \\
\hline Emergency caesarean section & 4 or $14.8 \%$ \\
\hline Elective caesarean section & 12 or $44 \%$ \\
\hline Scheduled caesarean section & 1 \\
\hline total & 27 \\
\hline
\end{tabular}

NB: data comparable with national average of $50-60 \%$ for mode of delivery. +Pediatrician present at delivery in 21 cases.

Table 28: Birth weight (grams).

\begin{tabular}{|c|c|}
\hline$<2500$ & 0 \\
\hline $2501-3000$ & 1 \\
\hline $3001-3500$ & 4 \\
\hline $3501-4000$ & $15^{\star}$ \\
\hline $4001-4500$ & 6 \\
\hline$>4500$ & 1 \\
\hline total & 27 \\
\hline
\end{tabular}

${ }^{*}$ GTT at 6 weeks postnatal period (GDM/IGT) 7/20 cases.

Unfavourable Outcomes (maternal and fetal)

Table 29: Maternal outcomes.

\begin{tabular}{|c|c|}
\hline PPH & $9^{*}$ \\
\hline DKA/hyperglycaemia & 2 \\
\hline Hypertension & 1 \\
\hline New end organ damage & 0 \\
\hline Worsening end organ damage & 0 \\
\hline Shoulder dystocia $^{\text {rd/4th }}$ degree tear & 0 \\
\hline Obstructed labour & 0 \\
\hline Maternal anaemia & 0 \\
\hline Maternal hyperglycaemia & 1 \\
\hline
\end{tabular}

with the national average of $50-60 \%$ of diabetic women delivered by caesarean section.

Table 28 shows that 15 babies had birth weight of 3501-4000g.

From the study it was discovered that only 7 out of 20 women who needed GTT 6 weeks postpartum actually had it. 
Table 30: Fetal outcomes.

Table 30: Fetal outcomes.
\begin{tabular}{|c|c|}
\hline SCBU admission & $17^{*}$ \\
\hline Neonatal hypoglycaemia & $5^{*}$ \\
\hline Neonatal jaundice & $9^{*}$ \\
\hline Hypothermia & 1 \\
\hline Respiratory distress & 2 \\
\hline Tube/poor feeding & 3 \\
\hline Phototherapy & 2 \\
\hline Resuscitation & 1 \\
\hline Neonatal infection & 2 \\
\hline
\end{tabular}

Adverse maternal outcome was mainly PPH (9 women), Table 29.

Fetal outcomes and morbidity are highlighted in table 30, the main ones are; SCBU admission, neonatal jaundice and hypoglycaemia. There was no fetal mortality. Some babies had more than one adverse outcome but there were no adverse fetal or maternal outcome in 7 cases.

The author had searched for the previous audits on diabetic pregnancies in the Hospital and was only able to find one audit performed 5 years earlier titled: Audit on Management of Diabetes in Pregnancies [6].

This audit was narrow in scope in that it looked at women with pre-existing diabetes and gestational diabetes mellitus (without significant details) booked for care at the Yeovil District Hospital during the period 01 January 2001 to 31 December 2001. The authors stated the standard they had used was based on the WHO's St. Vincent declaration. They stated they also used collated comparative data from national and regional audits which they used together with data the collated from teaching hospitals. This study whose content is about four pages of A4 papers in length, had only two tables, one on the type of labour and the other on type of delivery. There were no tables on fetal outcomes, some maternal outcomes and maternal features.

The women, the authors studied had age range of 24 to 41 with a mean of 30, all caucacians, with a BMI range of 20-30.5 with a mean of 24.8. The HbAlc ranged from 4.4 to 9.5 with a mean of 6.4 while birth weight ranged from 0.363 to 3.9 with a mean of $3 \mathrm{~kg}$.

Main findings of that audit were as follows: "Results from patients were included in this audit. $62.5 \%$ of women had type 1 diabetes, with $37.5 \%$ having gestational diabetes. Those women who had pre-existing diabetes had been diagnosed an average of 7 years. $37.5 \%$ of women were pregnant with their first child. $20 \%$ of women had received pre-pregnancy counselling".

It is clear the results were concerning and informative. The fact that the number of women studied was not stated made it hard to interpret the results, in the authors view. This author was unable to find out why as the lead author in the previous study could not be traced. There was also no section on "introduction" in the audit and "introduction section" is a very important opportunity for the authors to introduce the subject of diabetes; what their study was all about, and the scope and trajectory of their study.
That audit stated its Lower Segment Caesarean Section (LSCS) and complications as follows;

"LSCS and complications of the elective caesarean sections performed $50 \%$ were repeat caesarean sections. An emergency caesarean section was performed for cord presentation. There were a total of $50 \%$ obstetric complications.

$12.5 \%-\mathrm{PPH}+\mathrm{RPOC}+$ vaginal laceration

$12.5 \%$ - Cord presentation

$12.5 \%$ - Unstable lie

\section{$12.5 \%$ - Anti E antibodies}

$40 \%$ of women had episiotomies performed, with $20 \%$ having a laceration as a result of the birth.

Medical complications included $12.5 \%$ right posterior vitreous detachments. $25 \%$ of women had babies admitted to SCBU. Fetal complications included $12.5 \%$ with hypoglycaemia (at delivery) and $12.5 \%$ with orthopaedic problems."

These findings were revealing and informing. However, this present study stood superior and demonstrated more clarity and comprehension, and a clear departure from the previous study in many ways namely; it stated the number of women seen for a defined time frame and has 30 tables in its findings. This present study was able to state the target and study populations with a clear, reproducible and comprehensive methodology. It also has about 12 pages of information on the study and 2 pages of audit pro forma, with clearer and more comprehensive data, which would readily form an excellent template for future audits in this area in the department.

On recommendations, the authors of the previous audit recommended the following:

"Pre-pregnancy counselling to every diabetic woman with the involvement of GP

Diabetic team - Physicians and diabetic nurse (to include Obstetricians).

ANC - Ensure good communication and joint clinic at early stage.

Information leaflet for diabetes mellitus and gestational diabetes at clinics and GP surgeries.

Development of Protocol and Guidelines for hospital midwives for gestational and pre-pregnancy diabetes mellitus

GPs and Midwives to screen for gestational diabetes

Postnatal check in 6 weeks and GTT for women with gestational diabetes".

These recommendations were based on their findings and can easily be compared with the current recommendations (below) stated by this author which were also based on this author's findings. It is clear again that current study recommendations were clearer, more concise and more comprehensive.

\section{Recommendations}

(1) Pre-pregnancy care should be improved and optimised and it 
is the duty of the health care professionals who attend to these women before they become pregnant to encourage and support them to do so. It could also be that many women chose not to bother about the pre-pregnancy care.

(2) The quality of our surveillance of glycaemic control should be improved in line with the guidelines and acceptable standards in place, though we expect to have a Green Top guideline on Diabetic pregnancies in November 2007.

(3) Local protocol should be rehearsed (and updated) regularly in high risk antenatal clinic so that junior staff can comply better (and learn) with the outlined management plan.

(4) The number of women attending the High Risk Antenatal clinic should be managed in such a way that the attending staff can cope with the number of women attending the high risk antenatal clinic by recruiting more staff for the clinic or having two such clinics in a week, say on a Monday and on a Friday.

(5) It is recommended that staff that had been in the unit longer should be open to new scientific and evidence based information and practice and accept fresh evidence based inputs on management of these women. The guidelines and standard practice change from time to time as new evidence emerge. As other hospitals move to embrace evidence practice, so should our practice in this unit.

(6) Diabetic women should have clear information on the front of their hand-held hospital case notes detailing the necessary investigations and their timings during pregnancy, and also stating the need for strict compliance to the antenatal investigations and surveillance of glycaemic control.

\section{Conclusion}

Diabetic pregnancies will continue to fascinate the medical community for some time to come, as new scientific evidence continue to emerge to give a more detailed and clearer understanding of the extent of physiological, anatomical and biochemical aberrations, and alterations in diabetic pregnancies. This fascination is largely due to the effects of diabetes on pregnancy and also the effects of pregnancy on diabetes. It is widely known and agreed that without good glycaemic control, there is increased maternal, fetal and neonatal morbidity and mortality. How these effects play out depends on how the diabetes is managed and these in turn have geographical implications.
These effects are more severe in the developing countries, where essentially, achieving the objectives of St Vincent declaration (1012 October 1989), continue to be a dream or a mirage, compared to the developed countries, where achieving the objectives of this declaration is essentially or increasingly a reality.

Added to this is the increasing evidence of the hereditary implications of consanguinity in the development of type 2 diabetes; its geographical and ethnic spread; the extent and speed of derangement of glycaemic control and extent of complications $[3,4,9]$. The author speculates that as science advances, there could well be more clearer evidence that type 2 diabetes due to consanguinity could be worse than that not due to consanguinity and that these versions of type 2 diabetes could well be recognised and treated as distinct clinical entities.

\section{References}

1. Collin S, Arulkumaram S, Symonds IM, Hayes K, Jackson S, Impey L. Oxford Handbook of Obstetrics and Gynaecology. Oxford, Oxford University press. 2013; 3: 234-241.

2. Arulkumaram S, Symonds IM, Fowlie A. Oxford Handbook of Obstetrics and Gynaecology. Oxford, Oxford University press. 2004; 1: 190-194.

3. Bener A, Yousafzai MT, Al-Hamaq AO, Mohamad AG, DeFronzo RA Parental transmission of type 2 diabetes mellitus in a highly endogamous population. World J Diabetes. 2013; 4: 40-46.

4. Gosadi IM, Goyder EC, Teare MD. Investigating the Potential Effect of Consanguinity on Type 2 Diabetes Susceptibility in a Saudi Population. Hum Hered. 2014; 77: 197-206

5. Scottish Intercollegiate Guidelines Network, Scotland, UK. 2001

6. El-Sharafi $\mathrm{K}$ and Osoba O. Audit on Management of Diabetes in pregnancy, Department of Obstetrics and Gynaecology, Yeovil District Hospital, Yeovil Somerset, UK. 2002.

7. CEMACH Report, UK. 2002-2003.

8. CEMACH Report, UK. 2005.

9. Hadden D, Traub A, Lowy C, Nielsen GL, Sørensen HT, Olsen J, et al Outcome of pregnancy in women with Insulin dependent diabetes (1998) BMJ. 1998; 316: 550
Austin J Obstet Gynecol - Volume 4 Issue 1 - 2017 Submit your Manuscript | www.austinpublishing group.com Madu. (C) All rights are reserved
Citation: Madu AE. Diabetic Pregnancies in Yeovil, Somerset; Management and Outcomes. Austin J Obstet Gynecol. 2017; 4(1): 1069 